\title{
Autologous haematopoietic stem cell transplantation in juvenile idiopathic arthritis
}

\section{R Wedderburn, M Abinun, P Palmer, H E Foster}

\section{A powerful strategy when other treatments have failed}

$\mathrm{D}$ espite modern therapies, juvenile idiopathic arthritis (JIA) remains a significant cause of morbidity in children. The long term outcome of JIA is variable. Thus, while the prognosis for many children with JIA is good, over one third of children have ongoing active disease into adulthood with sequelae from chronic inflammation. ${ }^{12}$ Patients with systemic or polyarticular onset JIA tend to have a worse prognosis, even with the early use of disease modifying antirheumatic drugs (DMARDS). These patients have considerable morbidity from joint damage, osteoporosis, growth retardation, psychosocial morbidity, reduced quality of life, and educational or employment disadvantage. ${ }^{23}$

The concept of treating severe autoimmune disease by using intense, high dose immunosuppression to remove autoreactive lymphocytes, followed by rescue with haematopoietic stem cells, is based on the tenet that the immune system plays a pivotal role in such diseases. Support for this concept came from animal models, in which both spontaneous and induced autoimmune pathologies can be treated by lymphoablative/ myeloablative treatment followed by stem cell rescue. ${ }^{4}$ Early observations that chemotherapy treatment of malignancy in patients with concurrent autoimmunity could induce remission of autoimmune symptoms, led physicians to attempt haematopoietic transplantation for such disorders, initially in rheumatoid arthritis (RA), systemic lupus erythematosus (SLE,) and multiple sclerosis (MS). ${ }^{5}$

Abbreviations: ASCT, autologous stem cell transplantation; ATG, antithymocyte globulin; BMT, bone marrow transplantation; CMV, cytomegalovirus; DMARDs, disease modifying antirheumatic drugs; EBV, Epstein Barr virus; GVHD, graft versus host disease; G-CSF, granulocyte-colony stimulating factor; JIA, juvenile idiopathic arthritis; MAS, macrophage activation syndrome; MS, multiple sclerosis; NSCAG, National Specialist Commisionary Advisory Group; PBSC, peripheral blood stem cells; RA, rheumatoid arthritis; SLE, systemic lupus erythematosus; SOJIA, systemic onset JIA; $\mathrm{TBI}$, total body irradiation

1. Careful patient selection

4. Conditioning

5. Transplantation
In less than five years data have been collected on almost 400 patients who have undergone stem cell transplantation for autoimmune disorders. ${ }^{6}$ The majority have received autologous rather than allogeneic stem cells, since autologous transplantation carries lower mortality, and avoids the problems of graft matching and graft versus host disease (GVHD). Discussion continues about the potential curative nature of the use of allogeneic therapy, ${ }^{78}$ although some data suggest that arthritis can relapse even after allogeneic transplantation. ${ }^{9}$ This review concentrates on the role of autologous stem cell transplantation (ASCT) in the treatment of JIA; the steps are listed in table 1 . It is now widely thought that ASCT mediates a "resetting" of the immune system, rather than generating an entirely "new" immune system. Thus the use of stem cells can be seen as rescue during the period of immune depletion: this treatment is therefore sometimes known as autologous stem cell rescue (ASCR). Initial reports of the use of ASCT in children with severe JIA have been very encouraging. ${ }^{10}{ }^{11}$ To date over 45 ASCT procedures for JIA have been completed worldwide (N Wulffraat, personal communication). Since there is potential high morbidity and mortality associated with this procedure, it is imperative that children entered for ASCT are carefully selected, and that data are collated in order to allow standardisation of protocols.

\section{SELECTION OF PATIENTS}

Patient selection depends on three aspects: disease severity, failure of drug therapy, and drug toxicity. Since the realisation that JIA is not a benign disease, and that joint damage can occur early, paediatric rheumatologists have recognised that early aggressive treatment improves long term outcome. ${ }^{12}$ There has been an increasing trend of early use of intra-articular corticosteroids, and of methotrexate (MTX) as the DMARD of choice. MTX is often given parenterally to improve bioavailability and there is increasing evidence of efficacy in reducing joint damage. However, some patients respond poorly, develop toxicity or cannot tolerate MTX. For these children, therapeutic options have been limited to combination of DMARDs (for example, methotrexate with cyclophosphamide or cyclosporin), and more potent immunosuppression (for example, chlorambucil, or high dose/intravenous corticosteroids).

Despite the use of DMARDS, many children still receive oral steroids, with associated risks of growth retardation, osteoporosis, and cataracts. There are also concerns of long term effects including malignancy and infertility after chronic immunosuppression in children. ${ }^{13}$ In recent years new treatments including biological therapies have emerged. Lovell et al described dramatic improvement with etanercept (soluble TNFR, a TNF blocking agent) in children with polyarticular JIA refractory to methotrexate. ${ }^{14}$ However, in this study $28 \%$ of patients flared while taking sTNFR. The long term safety ${ }^{15}$ and efficacy of biological treatments is unknown, although initial reports, including use in adults for up to 43 months, suggest few long term side effects. ${ }^{16}{ }^{17}$ Thus the role of biological treatments is not yet clear. On this background, the availability of a novel, potentially curative, treatment for selected patients is welcome. ${ }^{18}$ Consensus guidelines for the use of ASCT in JIA, drawn up by the European Working party, ${ }^{19}$ and at an international meeting convened by the National Institutes of Health ${ }^{20}$ are summarised in table 2. In addition to careful clinical evaluation, the decision to proceed to ASCT must be with full informed consent of parents and, in an age appropriate manner, the patient. Risks and possible complications must be fully discussed, and weighed against those of

Table 1 Steps of an autologous stem cell transplant

2. Disease control prior to ASCT

3. Mobilisation, collection, and purification of stem cells

6. Supportive care (haematological and psychosocial)

7. Treatment and support after ASCT

8. Submission of information to international database 
Table 2 Inclusion and exclusion criteria for ASCT in JIA

\begin{tabular}{|c|c|}
\hline & Inclusion criteria \\
\hline 1. & Diagnosis of JIA according to ILAR criteria' \\
\hline 2. & Disease type systemic onset with polyarticular course, or polyarticular onset \\
\hline 3. & Disease duration at least one year \\
\hline 4. & $\begin{array}{l}\text { Evidence of active inflammatory disease for at least six months by accepted criteria, }{ }^{45} \text { but systemic patients must not have rash or high fever at } \\
\text { time of treatment }\end{array}$ \\
\hline \multirow[t]{6}{*}{5 . } & $\begin{array}{l}\text { Evidence of failure of, or toxicity from, standard therapy. Treatment failure includes patients whose disease is uncontrolled on: } \\
\text { High dose corticosteroids }(>0.3 \mathrm{mg} / \mathrm{kg}) \\
\text { High dose methotrexate }(1 \mathrm{mg} / \mathrm{kg}) \text { given parenterally for three months } \\
\text { Combination therapy of methotrexate and another drug, e.g. cyclosporin } \\
\text { Patients with active disease under treatment with "Seattle protocol" } 46 \text { for six months } \\
\text { Anti-TNF treatment given at appropriate doses for six months }\end{array}$ \\
\hline & Unacceptable toxicity includes: \\
\hline & Corticosteroid toxicity, e.g. growth failure, osteoporosis with fractures, avascular necrosis, hypertension, cataract \\
\hline & Unacceptable renal toxicity related to cyclosporin \\
\hline & Unacceptable elevation in liver enzymes related to methotrexate \\
\hline & Drug induced cytopenia \\
\hline 6. & Patient's general condition must be such that the procedure of ASCT itself will not pose an undue risk \\
\hline \multirow[t]{2}{*}{7.} & There is potential for improvement in functional disability \\
\hline & Exclusion criteria \\
\hline 1. & Severe chronic infection (particular caution with respect to TB) \\
\hline 2. & Severe functional disability with no potential for improvement \\
\hline 3. & Severe organ dysfunction (cardiac, respiratory, hepatic, renal) \\
\hline 4. & Inadequate social factors \\
\hline
\end{tabular}

conventional treatments, as well as quality of life issues, which may be different for each family.

\section{CONTROL OF ACTIVE DISEASE PRIOR TO ASCT}

The selection procedure for ASCT must ensure that disease is well controlled before this treatment is undertaken (table 2 ). The initial mortality rate in JIA patients treated by ASCT was significant, with four deaths among the first 29 patients. ${ }^{21}$ Deaths occurred from infection, and three had macrophage activation syndrome (MAS) associated with Epstein-Barr virus (EBV), adenovirus, and disseminated toxoplasmosis. ${ }^{21}{ }^{22}$ MAS is a well recognised and frequently fatal complication of SOJIA, but this number is above the expected rate for severe JIA. $^{23}$ Since these deaths may relate to severity of disease, the proposed selection criteria were amended. ${ }^{19}$ It was agreed that patients with active disease (in particular with persistent fevers) should not be offered ASCT until disease was controlled; immunosuppression after ASCT should be tapered slowly; and the graft must contain not less than $1-5$ $\times 10^{5} \mathrm{~T}$ cells $/ \mathrm{kg}$. The latter proposal remains controversial since the "threshold number" of $\mathrm{T}$ cells which can be reinfused without risking relapse is unknown.

\section{MOBILISATION AND STEM CELL PREPARATION}

Haematopoietic stem cells used in ASCT may be obtained either from bone marrow, or by inducing the exit of the cells into peripheral blood, known as mobilisation. Mobilisation is generally achieved using cyclophosphamide and granulocyte-colony stimulating factor (G-CSF) and is followed by apheresis, producing a high yield of peripheral blood stem cells (PBSC). Despite concerns that G-CSF may lead to flare of arthritis, ${ }^{6}$ this has not been reported in JIA. The use of PBSC avoids the need for a general anaesthetic, and produces a high yield of stem cells. This allows shorter periods of pancytopenia, and fewer complications from transfusions or infection..$^{24}$ Mobilisation itself has the potential benefit of the immunosuppressive effect of cyclophosphamide. Reports in adult RA have noted that some patients enter complete remission after mobilisation alone. ${ }^{26}{ }^{27}$ In children with JIA this effect has not yet been reported, although a small number of children with SLE have shown dramatic response after mobilisation (N Wulffraat, personal communication).

Once stem cells have been harvested they are purified, to remove mature lymphocytes. Much emphasis is placed on the level of $\mathrm{T}$ cell depletion required or achieved. Early studies of adult patients with malignancy and concurrent autoimmunity, whose bone marrow transplantation (BMT) was performed with non-T cell depleted marrow, strongly suggested that remission of autoimmune disease correlates with the removal of mature T cells. ${ }^{28}$ Two major strategies are employed to purify stem cells: negative selection which specifically depletes $\mathrm{T}$, or $\mathrm{T}$ and $\mathrm{B}$ cells, and positive selection of CD34+ stem cells. ${ }^{20}{ }^{29}$ Each can produce a 2-3 $\log _{10}$ fold reduction in T cell numbers. While full depletion of autoreactive $\mathrm{T}$ cells may be desirable, profound depletion is also associated with a higher risk of opportunistic infection including EBV and CMV reactivation, posttransplantation..$^{30}$ In addition, the belief that MAS in children with JIA is partly caused by uncontrolled activation by macrophages in the absence of $\mathrm{T}$ cell regulation led to a revision of the guidelines, suggesting that $\mathrm{T}$ cells should be at $1-5 \times 10^{5} \mathrm{~T}$ cells $/ \mathrm{kg} .{ }^{20}$ As yet there are no published data in JIA on whether outcome of ASCT correlates with degree of T cell depletion.

\section{CONDITIONING PRIOR TO TRANSPLANTATION}

Conditioning is generally performed 2-10 days prior to stem cell rescue and is designed to remove the cells mediating disease: many of the regimes used are highly immunosuppressive but not fully immunoablative or myeloablative. These regimens have been adapted from oncological practice: there is little known about how well they remove inflammatory cells from established sites of damage, such as the synovium. In adult RA one report has shown a reduction in inflammatory cells within the synovium. ${ }^{31}$ The most common combination used to condition JIA patients has been cyclophosphamide with antithymocyte globulin (ATG), and in some cases total body irradiation (TBI, 2 or 4 Gy). Other regimens have included busulphan, fludarabine, and etoposide ${ }^{1921}$ or other antibodies to lymphocytes. ${ }^{20} 32$ TBI remains controversial because of concerns about long term safety in children. It has been argued that long term effects of moderate dose TBI are no greater than those of conventional treatments of JIA, yet will allow a high success rate of ASCT. ${ }^{33}$ No trial has been performed to address this, but data submitted to the European Blood and 
Marrow Transplantation (EBMT) database suggest that children not given TBI have equally good outcome as those treated with irradiation.

\section{SUPPORTIVE CARE POST-ASCT}

ASCT is still considered an experimental treatment and is therefore reserved for those patients who have severe disease. ${ }^{34}$ The risk of death is significant, and the pretransplantation conditioning regimen has significant potential toxicity. Patients with chronic inflammatory diseases are at risk of opportunistic infections after ASCT because of previous long term and ongoing immunosuppression. Therefore appropriate screening for infections and meticulous surveillance is critical. $^{35}$ Immediately post-ASCT patients are managed as for pancytopenic states. The period of neutropenia is usually less than one month, and frequently less if PBSC are used. CD4 lymphopenia may be prolonged, and abnormalities of the $\mathrm{T}$ cell repertoire can last at least 12 months. ${ }^{32}$ Initially, rooms with laminar airflow and/or high efficiency particulate air (HEPA) filtration are generally used. Rigorous investigation of patients with fever, and immediate institution of treatment with broad spectrum antibiotics and/or antifungals is mandatory. Appropriate prophylaxis against Pneumocystis carinii, herpes viruses, and fungal infections is recommended, as well as regular intravenous immunoglobulin replacement until evidence of normal IgM production. In cases with unexplained fever $>39^{\circ} \mathrm{C}$ post-ASCT, in particular with a fall in platelet count or ESR, MAS should be considered. Treatment of MAS is with methylprednisolone $(20 \mathrm{mg} / \mathrm{kg} /$ day $)$ and cyclosporin $(2 \mathrm{mg} / \mathrm{kg} /$ day $)$. If no effect is seen within 48 hours, reinfusion of stored autologous $\mathrm{T}$ cells should be considered. ${ }^{20}$

\section{RESULTS TO DATE}

The data on 45 patients with JIA who have had ASCT in Europe are currently in preparation and show that approximately $50 \%$ of children show dramatic or "complete" remission of disease ( $\mathrm{N}$ Wulffraat, personal communication). Published data are available on 29 patients registered in the database of the Working Party for Autoimmune Diseases of the EBMT group, ${ }^{20}$ where the longest follow up was three years. Sixteen children were in "drug free remission", eight in partial remission or relapse, one was a non-responder, and four had died. As discussed above three deaths from MAS in the first 12 children treated with $\mathrm{T}$ cell depleted ASCT, led to alterations in the guidelines for patient selection, preparation, and management. To date there have been no further cases of MAS complicating ASCT in children with JIA. Nine of the 29 children still needed antirheumatic drugs. In adults with RA treated by ASCT it has been noted that as many as $40 \%$ may suffer some relapse of arthritis within a year, a rate that appears to be higher than in those children with JIA reported thus far. However, interestingly these adult patients had disease which was then easier to control with low doses of DMARDS, including drugs which had been previously ineffective pretransplantation. This observation adds weight to the concept that a "resetting" of the immune system may occur as a result of haematopoietic transplantation. The Dutch group of 14 children has been studied in detail. ${ }^{2036}$ Rheumatological data reveal that $7 / 14$ patients in remission showed more than 50\% improvement and 2/14 in partial remission a $30 \%$ improvement of their disease as measured by CHAQ score, physician global assessment, joint swelling/synovitis, and ESR. Younger children in particular had significant catch up growth, while this was less notable in older children with longer disease duration. Haematological reconstitution post-transplantation was uneventful, with neutrophils recovering to $>0.5 \times 10^{9} / 1$ by day +20 to +30 and platelets to $>20 \times 10^{9} / 1$ by day +16 to +35 . Two children had Staphylococcus epidermidis central venous catheter related infections. As in other patients treated with $\mathrm{T}$ cell depleted grafts, full immune function reconstitution was slow. The number of circulating $\mathrm{T}$ cells was normal at 6-9 months and in vitro proliferation to mitogens was normal by eight months post-ASCT. However CD4 lymphopenia $(<500 / \mathrm{ml})$ persisted for up to 12 months. Seven patients developed varicella zoster virus infection/reactivation as late as 18 months posttransplantation.

To date, there are no published data from children with JIA treated by ASCT which have addressed the issue of what proportion of the "new" lymphocyte repertoire has arisen from stem cells infused. From other studies it is known that reconstitution of full functional immunity after ASCT may take a year or more. ${ }^{37}$ The features and dynamics of immune function reconstitution after ASCT with T cell depletion studied in the Dutch group are similar to those seen after $\mathrm{T}$ cell depleted allogeneic BMT for severe combined immunodeficiency. ${ }^{38}$ Some data suggest that in the majority of haematopoietic transplants the resulting $\mathrm{T}$ and $\mathrm{B}$ cells are a mixture of either self and donor cells (in the allogeneic setting, so called chimerism), ${ }^{38}$ or "new" and "old" clones in the case of autologous transplantation. There is evidence that younger children have a higher proportion of new thymic emigrants-that is, T cells arising from new stem cells. This is likely to be a result of the greater remaining thymic function in young children. $^{40-42}$ One study of the $\mathrm{T}$ cell repertoire after ASCT treatment for a case of paediatric vasculitis, showed that $\mathrm{T}$ cell clones which arose rapidly in the CD45RO population just post-ASCT, were still detectable one year postASCT..$^{32}$ Thus an apparently normal T or B cell number can mask more subtle immunological deficiency, which may persist long after ASCT.

The initial cohort of JIA patients treated with ASCT had an unexpectedly high mortality rate (14\%), when compared to the usually quoted one of 3-5\% for ASCT. ${ }^{43}$ These patients may have other associated complications, such as pretransplantation disease related morbidity, drug related toxicity, long term immunosuppression, and potential conditioning toxicity. All these factors support the concept that ASCT with T cell depletion should only be performed in centres with transplantation expertise. $^{2043}$

\section{PSYCHOSOCIAL ASPECTS}

The transplantation procedure is complex and stressful. Awareness of these stresses at the outset can empower the family to cope with the lengthy transplantation process. ${ }^{44}$ The decision to opt for ASCT commits parents to a difficult journey of mixed emotions. The psychological turmoil caused by guilt, misgivings, and the desire to improve their child's quality of life makes huge demands on the family. There are two alternatives: to continue with conventional therapies or to consent to a novel treatment which can be lengthy and difficult, with a definite mortality risk, but which may result in improvement and even arrest of the disease process. The multidisciplinary team within the transplantation unit works with the family to ensure that an informed decision is reached. Through this decision making process the aim is to develop a trusting, open relationship, with both child and family. During this period the team identifies the family's coping strategies, common goals are agreed on, and supportive resources are identified and acquired. Strategies including home visits, clinic appointments, and play therapy can help the child and the family to familiarise themselves with the unit and its routines, help to overcome worries, and explore fears. The period of hospital stay is exhausting and challenging, but the family usually adapts well to the ward environment. The reinfusion of the stem cells is an important moment for the family, so everyone is encouraged to participate and record this special time. Preparation for discharge should be initiated prior to admission. The excitement of going home can be counteracted by the uncertainty of the result of transplantation. Getting back to "normal" 
and waiting for proven improvement can take time, and being well prepared prior to transplantation should minimise the fear during this time.

\section{PRACTICAL ASPECTS IN THE UK}

The procedure of ASCT, involving patient selection, the treatment itself, and subsequent follow up, involves a highly skilled multidisciplinary team. It is critical that patients are assessed by independent observers to avoid bias, and researchers should use the same protocols for patient selection and evaluation, so that results from different centres can be collated to evaluate the efficacy and safety of this new treatment. It is important that there is equitable access to treatment for all children with severe refractory disease, but also that expertise is concentrated in a few centres, to optimise outcome. In the UK, there are two centres with appropriate experience in dealing with children with severe immunodeficiencies, who can offer ASCT to children with JIA, namely Great Ormond Street Hospital for Children NHS Trust, London, and Newcastle upon Tyne Hospitals NHS Trust, Newcastle. These centres have been funded by the National Specialist Commisionary Advisory Group (NSCAG), with patients being referred for assessment for ASCT from paediatric rheumatologists within the UK, following the agreed guidelines. The British Paediatric Rheumatology Group (BPRG) has identified independent observers to assess these patients pre and post ASCT. Four UK paediatric rheumatology patients (two JIA, one JDM, and one PAN) have undergone ASCT so far, with good results. Information will be pooled with the EBMT database (http:// www.ebmt.org) to facilitate research and collective learning of this new and exciting treatment for refractory JIA.

\section{CONCLUSIONS}

Intense immunosuppression followed by lymphocyte depleted autologous stem cell rescue appears to be a powerful strategy for the control of severe juvenile arthritis after other treatments have failed, and one which can restore full function and activities to a child with previously devastating disease. The way forward now is for international collaboration, both in submission of data and comparison of protocols, and also for trials which set out to compare regimes for this approach to treatment. Collaborative studies to understand the results at a molecular level and how these relate to outcome are vital. In the future it is hoped that accurate and early prognostic indicators will be used to predict which child should receive intense immunosuppression and stem cell therapy early on, before the development of severe complications of the disease, such as growth retardation and joint damage.

\section{ACKNOWLEDGEMENTS}

We are grateful to the staff, patients, and parents at Great Ormond Street Hospital for Children and Newcastle General Hospitals, who have been vital participants in making ASCT a reality for children with JIA in the UK. We are very grateful to $\mathrm{Dr} \mathrm{N}$ Wulffraat (Utrecht, the Netherlands) for helpful discussions and advice, and for sharing data with us. This work was funded in part by grants from the Wellcome Trust and SPARKS (Sport Aiding Medical Research for Kids). This work was undertaken at Great Ormond Street Hospital for Children and Newcastle Hospitals NHS Trusts, which received a proportion of their funding from the UK NHS Executive; the views expressed in this publication are those of the authors and are not necessarily those of the NHS Executive. These centres have been funded by NSCAG for the use of ASCT in JIA patients. Finally we remember Dr Barbara Ansell for her inspiring lead in Paediatric Rheumatology.

\section{Arch Dis Child 2003;88:201-205}

\section{Authors' affiliations}

L R Wedderburn, Rheumatology Unit, Institute of Child Health, UCL and Great Ormond Street Hospital NHS Trust, London, UK

M Abinun, Department of Paediatric Immunology, Newcastle General Hospital, Newcastle Hospitals NHS Trust, Newcastle upon Tyne, UK

P Palmer, Children's Bone Marrow Transplantation Unit, Newcastle General Hospital, Newcastle Hospitals NHS Trust, Newcastle upon Tyne, UK

H E Foster, Departments of Rheumatology and Child Health, University of Newcastle upon Tyne and Newcastle Hospitals NHS Trust, Newcastle upon Tyne, UK

Correspondence to: Dr L R Wedderburn, Rheumatology Unit, Institute of Child Health, 30 Guilford Street, London WCIN 1EH, UK; l.wedderburn@ich.ucl.ac.uk

\section{REFERENCES}

1 Petty RE. Classification of childhood arthritis: a work in progress. Baillieres Clin Rheumatol 1998; 12:181-90

2 Zak M, Pedersen FK. Juvenile chronic arthritis into adulthood: a long-term follow-up study. Rheumatology (Oxford) 2000;39: 198-204.

3 Prieur AM, Chedeville G. Prognostic factors in juvenile idiopathic arthritis. Curr Rheumatol Rep 2001;3:371-8.

4 van Bekkum D. Immune ablation and stem-cell therapy in autoimmune disease. Experimental basis for autologous stem-cell transplantation. Arthritis Research 2000;2:281-4.

5 Tyndall A, Millikan S. Bone marrow transplantation. Baillieres Best Pract Res Clin Rheumatol 1999;13:719-35.

6 Tyndall A, Passweg J, Gratwohl A. Haemopoietic stem cell transplantation in the treatment of severe autoimmune diseases 2000. Ann Rheum Dis 2001;60:702-7.

7 McSweeney P. Nonmyeloablative allogeneic haematopoietic cell transplants: any role for rheumatoid arthritis? J Rheumatol 2001;28(suppl 64):49-54

8 Burt RK, Barr W, Oyama Y, et al. Future strategies in haematopoietic stem cell transplantation for rheumatoid arthritis. Rheumatol 2001;28(suppl 64):42-8.

9 Snowden JA, Kearney P, Kearney A, et al. Long-term outcome of autoimmune disease following allogeneic bone marrow transplantation. Arthritis Rheum 1998;41:453-9.

10 Wulffraat N, van Royen A, Bierings $M$, et al. Autologous haemopoietic stem-cell transplantation in four patients with refractory juvenile chronic arthritis. Lancet 1999;353:550-3.

11 Nakagawa R, Kawano Y, Yoshimura E, et al Intense immunosuppression followed by purified blood CD34+ cell autografting in a patient with refractory juvenile rheumatoid arthritis. Bone Marrow Transplant 2001;27:333-6

12 Wallace CA, Levinson JE. Juvenile rheumatoid arthritis: outcome and treatment for the 1990s. Rheum Dis Clin North Am 1991;17:891-905.

13 Laxer RM. Long-term toxicity of immune suppression in juvenile rheumatic diseases. Rheumatology (Oxford) 1999;38:743-6.

14 Lovell DJ, Giannini EH, Reiff A, et al. Etanercept in children with polyarticular juvenile rheumatoid arthritis. Paediatric Rheumatology Collaborative Study Group. N Engl J Med 2000;342:763-9.

15 Myers A, Clark J, Foster H. Tuberculosis and treatment with infliximab. N Engl J Med 2002;346:623-6.

16 Moreland LW, Cohen SB, Baumgartner SW, et al. Long-term safety and efficacy of etanercept in patients with rheumatoid arthritis. J Rheumatol 2001;28:1238-44.

17 Takei S, Groh D, Bernstein B, et al. Safety and efficacy of high dose etanercept in treatment of juvenile rheumatoid arthritis. $J$ Rheumatol 2001;28:1677-80.

18 Petty RE. Prognosis in children with rheumatic diseases: justification for consideration of new therapies. Rheumatology (Oxford) 1999;38:739-42.

19 Wulffraat NM, Kuis W, Petty R. Addendum: proposed guidelines for autologous stem cell transplantation in juvenile chronic arthritis. Paediatric Rheumatology Workshop. Rheumatology (Oxford) 1999;38:777-8.

20 Barron KS, Wallace C, Woolfrey CEA, et al. Autologous stem cell transplantation for pediatric rheumatic diseases. J Rheumatol 2001;28:2337-58

21 Wulffraat NM, Kuis W. Treatment of refractory juvenile idiopathic arthritis. $J$ Rheumatol 2001;28:929-31.

22 Quartier P, Prieur AM, Fischer A. Haemopoietic stem-cell transplantation for juvenile chronic arthritis. Lancet 1999;353: 1885-6.

23 Sawhney S, Woo P, Murray KJ. Macrophage activation syndrome: a potentially fatal complication of rheumatic disorders. Arch Dis Child 2001;85:421-6.

24 Schmitz N, Linch DC, Dreger P, et al. Randomised trial of filgrastim-mobilised peripheral blood progenitor cell transplantation versus autologous bone-marrow transplantation in lymphoma patients. Lancet 1996;347:353-7.

25 Figuerres E, Haut PR, Olzewski $M$, et al. Analysis of parameters affecting engraftment in children undergoing autologous peripheral blood stem cell transplants. Bone Marrow Transplant 2000;25:583-8.

26 Breban $M$, Dougados $M$, Picard $F$, et al. Intensified-dose $(4 \mathrm{gm} / \mathrm{m} 2)$ cyclophosphamide and granulocyte colony-stimulating factor administration for haematopoietic stem cell mobilisation in refractory rheumatoid arthritis. Arthritis Rheum 1999;42:2275-80.

27 Burt RK, Fassas A, Snowden J, et al. Collection of haematopoietic stem cells from patients with autoimmune diseases. Bone Marrow Transplant 2001;28:1-12.

28 Euler HH, Marmont AM, Bacigalupo A, et al. Early recurrence or persistence of autoimmune diseases after unmanipulated autologous stem cell transplantation. Blood 1996:88:3621-5.

29 Slaper-Cortenbach IC, Wijngaarden-du Bois M, de Vries-van Rossen A, et al. The depletion of T cells from haematopoietic stem cell transplants. Rheumatology (Oxford) 1999:38:751-4.

30 Holmberg LA, Boeckh M, Hooper $\mathrm{H}$, et al.. Increased incidence of cytomegalovirus 
disease after autologous CD34-selected peripheral blood stem cell transplantation. Blood 1999;94:4029-35.

31 Fearon U, Bingham S, Mewar D, et al. Analysis of TNF, NFkB and MMP-1 expression in resistant RA pre and post autologous stem cell transplantation. Arthritis Rheum 2001;44:S214

32 Wedderburn LR, Jeffery $R$, White $H$, et al. Autologous stem cell transplantation for paediatric-onset polyarteritis nodosa: changes in autoimmune phenotype in the context of reduced diversity of the T- and B-cell repertoires, and evidence for reversion from the $\mathrm{CD} 45 \mathrm{RO}(+)$ to $\mathrm{RA}(+)$ phenotype. Rheumatology (Oxford) 2001 :40:1299-307.

33 van Bekkum DW. Effectiveness and risks of total body irradiation for conditioning in the treatment of autoimmune disease with autologous bone marrow transplantation. Rheumatology (Oxford) 1999;38:757-61.

34 Ilowite NT. Current treatment of juvenile rheumatoid arthritis. Pediatrics 2002; 109:109-15

35 Dini G Castagnola E Comoli P, et al. Infections after stem cell transplantation in children: state of the art and recommendations. Bone Marrow Transplant 2001;28(suppl 1):S18-21.

36 Wulffraat N, Kamphuis SSM, van der Net J et al. Autologous haemopoietic stem cell transplantation in 12 cases with refractory juvenile idiopathic arthritis. Osteological Bulletin 2000:2:62-5.

37 Guillaume T, Rubinstein DB, Symann M. Immune reconstitution and immunotherapy after autologous haematopoietic stem cell transplantation. Blood 1998;92:1471-90.

38 Gennery A, Dickinson A, Brigham K, et al. CAMPATH 1M T cell depleted BMT for SCID: long-term follow-up of 19 children treated 1987-98 in a single centre. Cytotherapy $2001 ; 3: 221-32$.

39 Haddad E, Le Deist F, Aucouturier P, et al. Long-term chimerism and B-cell function after bone marrow transplantation in patients with severe combined immunodeficiency with $B$ cells: a single-centre study of 22 patients. Blood 1999;94:2923-30.

40 Mackall CL, Fleisher TA, Brown MR, et al. Age, thymopoiesis, and CD4+ T-lymphocyte regeneration after intensive chemotherapy. $N$ Engl J Med 1995:332:143-9.

41 Patel DD, Gooding ME, Parrott RE, et al. Thymic function after haematopoietic stem-cell transplantation for the treatment of severe combined immunodeficiency. N Engl J Med 2000;342: 1325-32.

42 Myers LA, Patel DD, Puck JM, et al. Haematopoietic stem cell transplantation for severe combined immunodeficiency in the neonatal period leads to superior thymic output and improved survival. Blood 2002:99:872-8.

43 Laxer, RM, Harrison C. Bioethical issues in autologous stem cell transplantation in children and adults with arthritis. J Rheumatol 2001:28:2147-50.

44 Wochna V. Anxiety, needs, and coping in family members of the bone marrow transplant patient. Cancer Nurs 1997:20:244-50.

45 Giannini EH, Ruperto N, Ravelli A, et al. Preliminary definition of improvement in juvenile arthritis. Arthritis Rheum 1997:40:1202-9.

46 Wallace CA, Sherry DD. Trial of intravenous pulse cyclophosphamide and methylprednisolone in the treatment of severe systemic-onset juvenile rheumatoid arthritis. Arthritis Rheum 1997;40:1852-5.

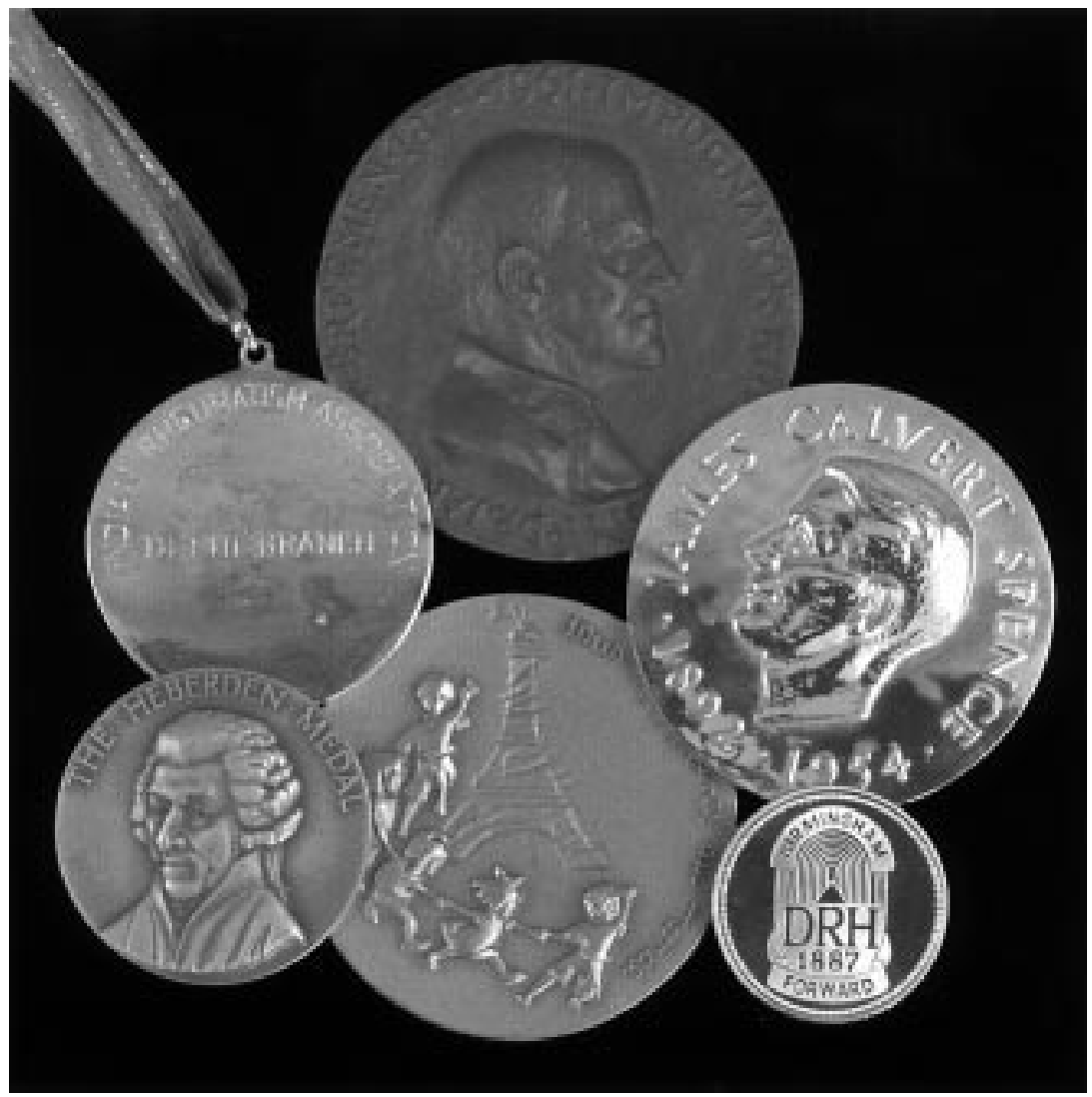

Barbara Ansell's medals 